\title{
A Research on Controlling Topology of Wireless Sensor Networks Against Node Based Attacks
}

\author{
M. Hari Kishan Rao, M.Pushpalatha
}

\begin{abstract}
The topology design with survivability and reliability is a crucial aspect in wireless-sensor networks. Controlling the topology is very important as it can affect the efficiency of Wireless-Sensor Networks (WSN).To address the problem, an approach on energy efficiency is proposed on scale free networks. The nodes in the network are evenly distributed to accommodate scaling of the network. The quality parameters like energy and load balance should be captured by the network, as factors like residual energy of the node and node degree are taken into consideration. Arrangements are made to look at the network in a practical way and core functionality of the network is not disturbed. Strategies are deployed to make the network robust for node based random and malicious attacks. In this Survey, we concentrate on the study of topology control techniques with energy based parameters, change the topology of the network to improve efficiency of network in terms of network lifetime.
\end{abstract}

Keywords- Scale free Networks, Node attacks, Topology control, Lifetime of the network, Wireless Sensor Networks.

\section{INTRODUCTION}

WSNs are combination of sensor nodes connected together and do not have a fixed infrastructure. Sensor nodes have a processing unit with some power and energy. These are deployed in various regions to read the environmental parameters and monitor them. They are created to be used in various fields like defense, health care, commercial and industrial applications. These can be extended for real time applications to monitor various synchronizations, processing, storing, discovering etc. Typically many autonomous devices like end nodes and some devices combine to form gateway like structure to facilitate bridging, buffering from wireless network to Ethernet network. Wireless Sensor Networks (WSN) are efficient to monitor and collect the data compared to only communications in autonomous systems[1].The routing and network communication protocols will have major effect on lifetime of network, consumption of energy and data latency. Security became the biggest threat in recent days as the number of cyber-attacks are increasing day by day, the network should be able to withstand the attacks and stay secure until the data transmission ends. The scale free networks are used to model homogeneous network topologies. Thus, the nodes have very similar degrees and will be used to get rid of random attacks on the network. In this paper, we are only interested in only conserving the topology of the

Revised Manuscript Received on April 12, 2019.

M. Hari Kishan Rao, Department of Computer Science and Engineering, SRM University, Kattankulathur, Kancheepuram District, Tamil Nadu -603103. India (E-mail: harikishanm123@gmail.com)

Dr.M.Pushpalatha, Department of Computer Science and Engineering, SRM University, Kattankulathur, Kancheepuram District, Tamil Nadu -603103. India
WSN's[2][3]. The degree distribution between the nodes will play major role in the robustness of the network, the nodes with dissimilar degrees shows stronger tendency towards each other than nodes with same degree[4][5].

\section{SCALE FREE NETWORKS}

The Random network has nodes with almost equal number of connections for the each node and are scaled to certain limit where the distribution of the links are limited. But practically we are working with some nodes with links more than hundred links and few nodes need not have many links. This study of random network inspires us to make scale free networks that follow power law of distribution. Hubs are nodes with high number of links around them communicating with the other nodes. This hubs concept came into existence with the introduction of the Scale free networks. The advantages of using Scale free networks outweighs the advantages of random networks. So, we move from random networks to scale free networks. Both the networks are in for a single purpose but the way the formation of nodes and their interconnections are different.

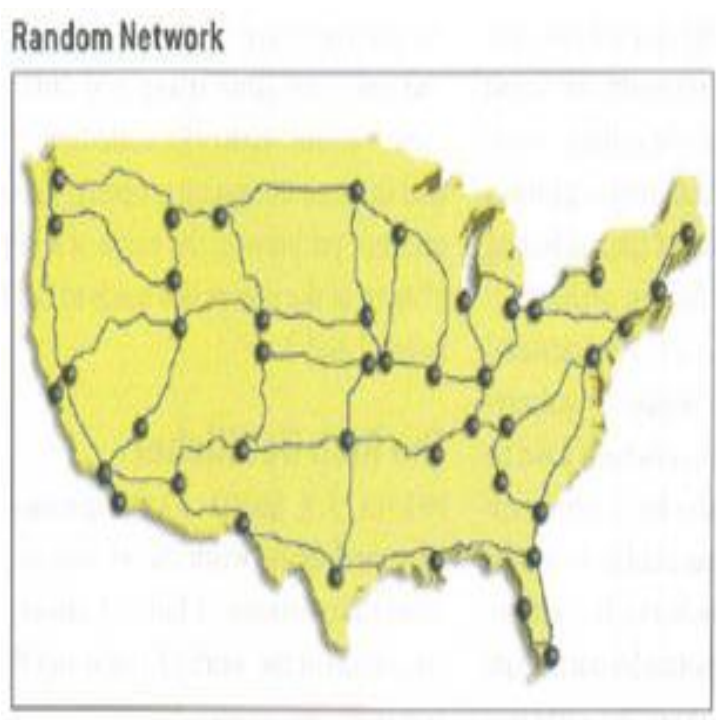

Figure 1- Random Network. 
Scale-Free Network

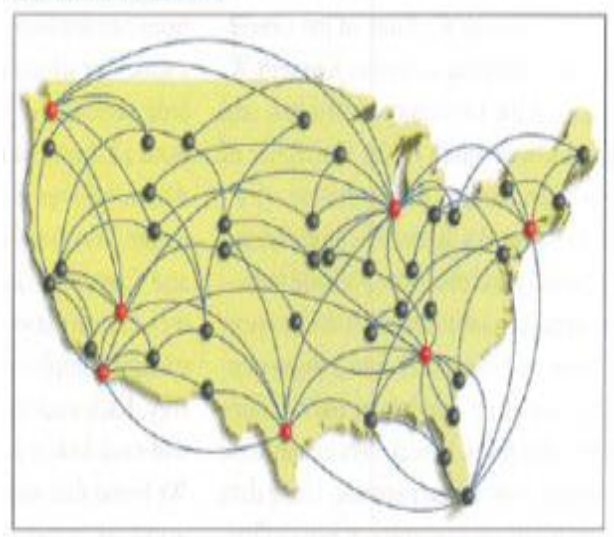

Figure 2- Scale Free Network.

\section{Power Law Distribution of Node Linkages}

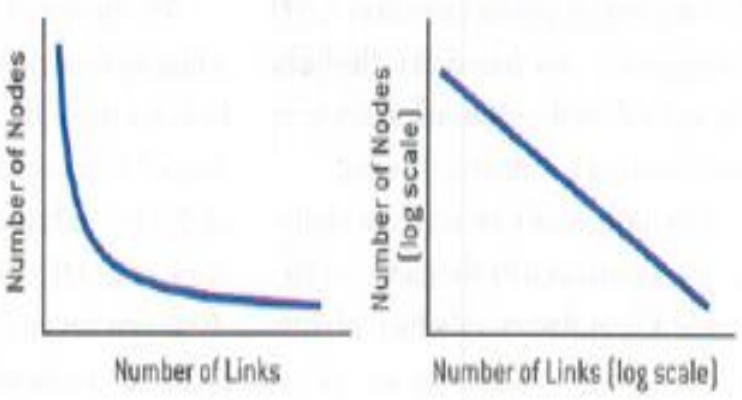

Figure 3- Power Law Distribution

The Scaling of the network means changing the topology of the network as it should be robust to various node based attacks. It is adding nodes progressively to nodes already in the network with some parameter making decision on attachment of the node to other[6]. The best example of scale free network is network(Internet) with routers as nodes and links as optical connections.

Fig 1.1 shows red colored nodes a hubs as they have many number of connections and others are normal nodes with similar connections. The Hubs are connected to different nodes irrespective of distance between them.

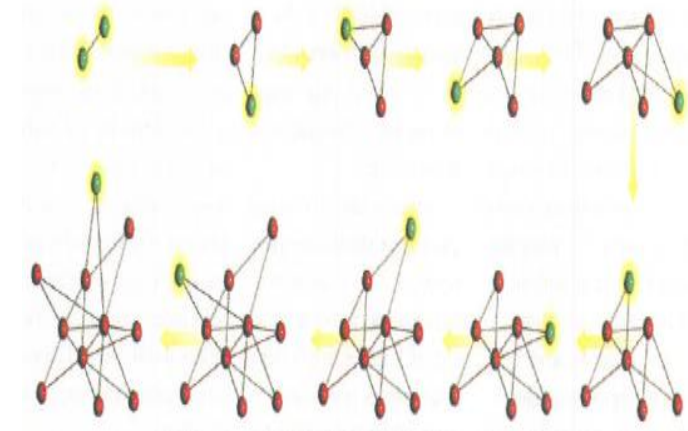

Figure 4- Scale Free Network building.

The Figure gives basic idea of architecture of scale free network. The nodes red in color are existing nodes and nodes green in color are recently added node to the network. The Hubs in the network will dominate the network with basic mechanisms like growth and preferential attachment [7].

\section{TOPOLOGY OF THE NETWORK \& RESULTS}

Topology of a network has an important role to play in the life time and efficiency of the network as the scale free efficient topology is gaining more importance as they are robust to node based attacks[8]. The failure of the certain node in any network will disconnect the neighboring nodes to the network. Upon attack, the network should be able to transfer its responsibilities to another node based degree and similarity of the node to the other. So, the node failure should not be affecting the functionality of the network. Without study of energy parameters of the node, it is difficult to know when the node failure occurs and will be serious drawback in terms of functionality of the network as the functional node failure make network disconnected. Controlling the topology of the network means giving control of changing few parameters of the network by the sensor nodes and form a different structure based on the need[9].The sensor nodes can discover its neighboring nodes of the network by its transmission power. The ultimate goal of topology control is to maintain a topology with less energy consumption and high efficiency. Clustering the set of nodes is helpful in topology control as it can control the set of neighboring nodes in the cluster.

Security of a network has vital importance as the attackers try to break the network with different intensions. The attacks on the node are increasing day by day as the importance of data is. The attacks will be different for different layers in OSI mode. The data security is important in every layer irrespective of importance. In this paper, we discuss about Random and Targeted attacks on the node. Random attacks are attacks which chooses any node in the network randomly and attacks it. The scale free topology is very efficient to random attacks as only nodes with low degree will be attacked and functional nodes are less likely to be targeted [10]. The nodes with low degree does not affect the performance of the network[16]. Targeted attacks are attacks on specific node with key functionality that form the core of the network. The drop in the packets of the network or congestion means that the network is attacked and need to deploy any strategy to solve the problem. There are very specific and dangerous attacks as they have particular motive of targeting the key nodes. The functional nodes should be protected from the malicious attacks to make the network robust against attacks. The scale free topology is not proven to be formidable against the malicious attacks. So we make the network form onion like structure and protect the functional nodes form attack. The operations like Degree difference operation and Angle sum operation are performed to scale the network like a structure like onion[10]. The onion structure is very standard and can withstand malicious attacks by the hacker to build a topology which is free from functional node failures. In the network the topology is arranged in such a way that the nodes with higher degree are at center surrounding with low degree nodes. 


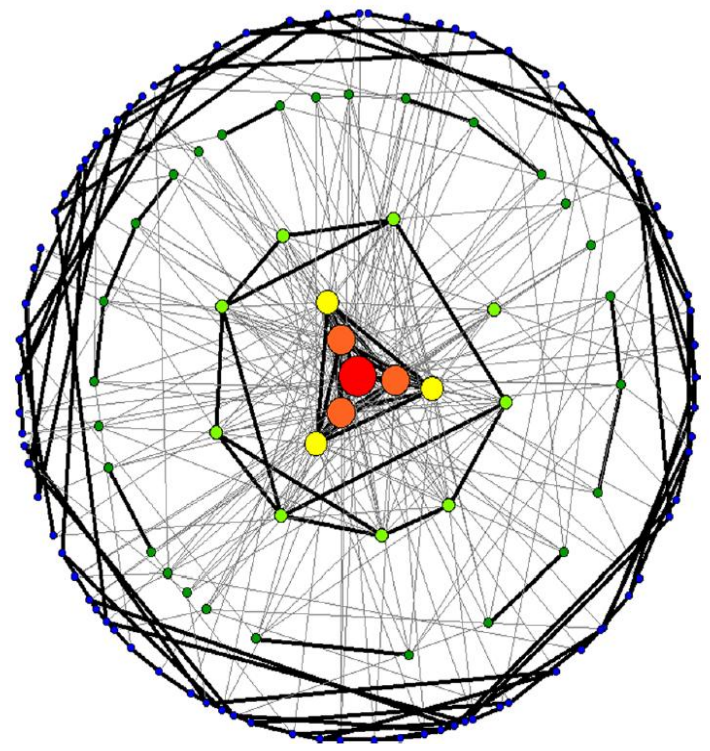

Figure 5- Onion Like structure

\section{ENERGY OF THE NODE}

The sensors with high energy are bound to run for longer period of time. The energy wastage problem should be addressed as the efficiency of the network get affected with more wastage of the energy.

The topology of the networks will be specific to particular application. The energy of the nodes depends on the requirement of the network and not fixed to any parameter. There are many approaches to energy saving of the node but none of the approaches made other approaches irrelevant. It means, we do not have universally acceptable energy efficient algorithm[11].V. Rodoplu, T. H. Meng[12] proposed an algorithm on Minimum Energy Communication Network (MECN).This uses the concept of relay transmission to develop a topology of paths with low energy and transmit to any wireless sensor network to sink node[12].First the node gets set of nodes it can communicate within its reach and makes sure the energy consumption is also less. Then uses shortest path technique to get path with minimum energy to the sink node.

The energy efficiency primarily consists of coverage area, spreading request and data aggregation[13][14].In [15] the error bounds of the sensor nodes are dynamically allocated and measured the data aggregation in terms of lifetime of a network.

\section{CONCLUSION}

The topology control is very important aspect of wireless sensor networking because of its change in the node position, the efficiency of the network can be improved. Without disturbing the power law distribution between the nodes, we can make the network approach the onion like structure to be efficient enough to tackle malicious attacks on the nodes. The topology control with energy efficient factors like load and energy balance are taken into account while designing the network. By this method we can improve the efficiency of the network in terms of throughput and lifetime of the network.

\section{ACKNOWLEDGEMENT}

I sincerely thank my University and staffs who contributed to and helped in this research work. This would not have been here without them.

\section{REFERENCES}

1. Ze Li and Haiying shen, "A QoS-oriented distributed routing protocol for hybrid wireless networks," IEEE Transactions on Mobile Computing, March 2014.

2. Reka Albert, Hawwong Jeong and Albert Laszlo Barabasi "Error and attack tolerance of the complex networks"27 July, 2000.

3. Salvatore Scellato, Ilias Leontiadis, C Mascolo, Prithwish Basu "Evaluating the temporal robustness of mobile networks" IEEE Transactions on Mobile Computing ,January 2013.

4. Mingjing Zhou, Jinh Liu "A memetic algorithm for enhancing the robustness of scale-free networks against malicious attacks" Statistical mechanics and applications, Sept 2014.

5. M. E. J. Newman,"Assortive mixing in Networks" Phys. Rev. Lett. 89, 208701 (2002).

6. Ming-Yang-Wang, Guang Yu, Da Ren-Yu. "Scale free model for wireless sensor networks" IEEE International Conference on Intelligent Computing and Intelligent Systems.

7. D'Angelo and Stefano Ferretti."'Simulation of scale free Networks" Published in Second International Conference on Simulation Tools and Techniques (SIMUTools 2009).

8. Gengzhong Zheng, Sanyang Liu and Xiaogang Qi "Scale-free topology evolution for WSN's with reconstruction mechanism". Published in May 2012.

9. Bharadhwaj and Chandrashekaran, "Low powered Wireless Sensor Networks", Published in VLSI Design 2001. Fourteenth International Conference on VLSI Design.

10. Tie Qiu, Zhao, Xia, Weisheng, Dapeng. "ROSE: Robustness Strategy for Scale-Free Wireless Sensor Networks". Published in IEEE/ACM Transactions on Networks in 2017, October.

11. Zaman, Tang Jung, Mehboob Yasin "Energy Efficiency in Wireless Sensor Networks", Journal of sensors 2016.

12. Volkan Rodpolu Teresa H. Meng, "Minimum energy mobile wireless networks.Published on Aug 1999 in IEEE.

13. TVPadmavathy" Extending Network Lifetime Using Optimized Energy Efficient Cross Layer Module (OEEXLM) in Wireless Sensor Network",2009 April,

14. Ruihua, Zhiping and Dongfeng, "Analysis of Lifetime of Large Wireless Sensor Networks Based on Multiple Battery Levels", Published on May, 2008 in SciRes.

15. Xueyan Tang and Jianliang Xu, Extending Network Lifetime for Precision-Constrained Data Aggregation in Wireless Sensor Networks, IEEE Communication Society, 2006.

16. S Lee, B Bhattacharjee, S Banarjee and B Han,"A General framework for efficient Geographic routing in Wireless Networks", AT\&T Labs. 